\title{
An Optimal Portfolio and Capital Management Strategy for Basel III Compliant Commercial Banks
}

\author{
Grant E. Muller and Peter J. Witbooi \\ University of the Western Cape, Private Bag X17, Bellville 7535, South Africa \\ Correspondence should be addressed to Peter J. Witbooi; pwitbooi@uwc.ac.za
}

Received 3 October 2013; Accepted 5 January 2014; Published 19 February 2014

Academic Editor: Francesco Pellicano

Copyright (C) 2014 G. E. Muller and P. J. Witbooi. This is an open access article distributed under the Creative Commons Attribution License, which permits unrestricted use, distribution, and reproduction in any medium, provided the original work is properly cited.

\begin{abstract}
We model a Basel III compliant commercial bank that operates in a financial market consisting of a treasury security, a marketable security, and a loan and we regard the interest rate in the market as being stochastic. We find the investment strategy that maximizes an expected utility of the bank's asset portfolio at a future date. This entails obtaining formulas for the optimal amounts of bank capital invested in different assets. Based on the optimal investment strategy, we derive a model for the Capital Adequacy Ratio (CAR), which the Basel Committee on Banking Supervision (BCBS) introduced as a measure against banks' susceptibility to failure. Furthermore, we consider the optimal investment strategy subject to a constant CAR at the minimum prescribed level. We derive a formula for the bank's asset portfolio at constant (minimum) CAR value and present numerical simulations on different scenarios. Under the optimal investment strategy, the CAR is above the minimum prescribed level. The value of the asset portfolio is improved if the CAR is at its (constant) minimum value.
\end{abstract}

\section{Introduction}

Successful bank management can be achieved by addressing four operational concerns. Firstly, the bank should be able to finance its obligations to depositors. This aspect of bank management is called liquidity management. It involves the bank acquiring sufficient liquid assets to meet the demands from deposit withdrawals and depositor payments. Secondly, banks must engage in liability management. This aspect of bank management entails the sourcing of funds at an acceptable cost. Thirdly, banks are required to invest in assets that have a reasonably low level of risk associated with them. This process is referred to as asset management. It aims to encourage the bank to invest in assets that are not likely to be defaulted on and to adopt investment strategies that are sufficiently diverse. The fourth and final operational concern is capital adequacy management. Capital adequacy management involves the decision about the amount of capital the bank should hold and how it should be accessed. From a shareholder's perspective, utilizing more capital will increase asset earnings and will lead to higher returns on equity. From the regulator's perspective, banks should increase their buffer capital to ensure the safety and soundness in the case where earnings may end up below an expected level. In this paper, we address problems associated with asset and capital adequacy management.

The Basel Committee on Banking Supervision (BCBS) regulates and supervises the international banking industry by imposing minimal capital requirements and other measures. The 1998 Basel Capital Accord, also known as the Basel I Accord, aimed to assess the bank's capital in relation to its credit risk, or the risk of a loss occurring if a party does not fulfill its obligations. The Basel I Accord launched the trend toward increasing risk modeling research. However, its oversimplified calculations and classifications have simultaneously called for its disappearance. This paved the way for the Basel II Capital Accord and further agreements as the symbol of the continuous refinement of risk and capital. The 2004 (revised) framework of the Basel II Capital Accord (see [1]) laid down regulations seeking to provide incentives for greater awareness of differences in risk through more risk-sensitive minimum capital requirements based on numerical formulas. The Total Capital Ratio or Capital Adequacy Ratio (CAR) (see for instance [2-6]) measures the amount of the bank's capital relative to its amount of credit exposures. Internationally, a standard has been adopted 
that requires banks to adhere to minimum levels of capital requirements. By complying with minimum capital levels, banks are guaranteed the ability to absorb reasonable levels of losses before becoming insolvent. Thus, CARs ensure the safety and stability of the banking system. Under the Basel II Accord, banks were required to maintain a CAR, that is, a ratio of total bank capital to total risk-weighted assets (TRWAs), with a minimum value of $8 \%$.

In response to the 2007-2008 financial crisis, the Basel Committee on Banking Supervision (BCBS) introduced a comprehensive set of reform measures known as the Basel III Accord. The Basel III Accord is aimed at improving the regulation, supervision, and risk management within the banking sector and is part of the continuous effort made by the BCBS to enhance the banking regulatory framework. The Basel III Accord builds on the Basel I and II documents and seeks to improve the banking sector's ability to deal with financial and economic stress, improve risk management, and strengthen the banks' transparency. The focus of the Basel III Accord is to foster greater resilience at the individual bank level in order to reduce the risk of system wide shocks. In this regard, the Basel III Accord contains changes in the following areas: (i) augmentation in the level and quality of capital; (ii) introduction of liquidity standards; (iii) modifications in provisioning norms; (iv) introduction of a leverage ratio. For a detailed discussion on the aforementioned enhancements in the Basel III Accord over the Basel II Accord, see the paper [7] of Jayadev or the book [8] of Petersen and MukuddemPetersen.

In our paper, we are concerned with the level of bank capital. Thus we study the Total Capital Ratio or CAR which, if denoted by $\Lambda$, is defined as

$$
\Lambda=\frac{C}{a_{\mathrm{rw}}} .
$$

In the expression for the CAR above, $C$ denotes the total bank capital and $a_{\mathrm{rw}}$ the TRWAs of the bank. Under Basel III rules, the minimum prescribed value of the bank's CAR remains unchanged at $8 \%$. However, banks are now required to hold a Capital Conservation Buffer (CCB) of $2.5 \%$ and Countercyclical Buffer Capital (CBC) in the range of 0$2.5 \%$. The CCB ensures that banks can absorb losses without breaching the minimum capital requirement and are able to carry on business even in a downturn without deleveraging. The $\mathrm{CBC}$ is a preemptive measure that requires banks to build up capital gradually as imbalances in the credit market develop. It may be in the range of $0-2.5 \%$ of risk-weighted assets which could be imposed on banks during periods of excess credit growth. There is also a provision for a higher capital surcharge on systematically important banks (see [7, 8]).

In finance, the stochastic optimal control method is a popular optimization technique and was used for the first time by Merton [9, 10]. This technique involves solving the Hamilton-Jacobi-Bellman (HJB) equation arising from dynamic programming under the real world probability measure. Examples of the application of this technique in the banking literature can be the papers by MukuddemPetersen and Petersen [11], Mukuddem-Petersen et al. [12],
Mukuddem-Petersen et al. [13], and Gideon et al. [14] for instance. In their paper, Mukuddem-Petersen and Petersen [11] studied a problem related to the optimal risk management of banks in a stochastic setting. The aforementioned authors minimized market and capital adequacy risk that respectively involves the safety of the securities held and the stability of sources of funds. In this regard, the authors suggested an optimal portfolio choice and rate of bank capital inflow that will keep the loan level as close as possible to an actuarially determined reference process. In the paper [12] by Mukuddem-Petersen et al., the authors solved a stochastic maximization problem that is related to depository consumption and banking profit on a random time interval. In particular, they showed that it is possible for a bank to maximize its expected utility of discounted depository consumption on a random time interval, $[t, \tau]$, and its final profit at time $\tau$. In the paper [13] of Mukuddem-Petersen et al. (see also the book of Petersen et al. [15]) the authors analyzed the mortgage loan securitization process which was the root cause of the subprime mortgage crisis (SMC) of 2008. The authors considered a bank that has the cash outflow rate for financing a portfolio of mortgage-backed securities (MBSs) and studied an optimal securitization problem with the investment in the MBSs as controls. Gideon et al. [14] quantitatively validated the new Basel III liquidity standards as encapsulated by the net stable funding ratio. By considering the inverse net stable funding ratio as a measure to quantify the bank's prospects for a stable funding over the period of a year, they solved an optimal control problem for a continuous-time inverse net stable funding ratio. More specifically, the authors made optimal choices for the inverse net stable funding targets in order to formulate its cost. The latter was achieved by finding an analytical solution for the value function.

In theoretical physics, the Legendre transform is commonly used in areas such as classical mechanics, statistical mechanics, and thermodynamics. The Legendre transform also has its uses in mathematics of finance. For instance in pension fund optimization problems, the Legendre transform is generally applied to transform nonlinear HJB PDEs arising from the optimal control technique to linear PDEs for which it is easier to find a closed form solution. Authors who have applied the Legendre transform in pension funds are Xiao et al. [16] on the constant elasticity of variance (CEV) model for a defined contribution pension plan and the Legendre transform-dual solutions for annuity contracts, Gao [17] on the stochastic optimal control of pension funds, Gao [18] on the optimal investment strategy for annuity contracts under the CEV model, Gao [19] on the extended CEV model and the Legendre transform-dual-asymptotic solutions for annuity contracts, and Jung and Kim [20] on the optimal investment strategies for the hyperbolic absolute risk aversion (HARA) utility function under the CEV model.

In our contribution, the issues of asset portfolio and capital adequacy management for Basel III compliant commercial banks are addressed in a continuous-time setting. More specifically, our goal is to maximize an expected utility of a commercial bank's asset portfolio at a future date. We assume that the interest rate in the market is stochastic and that the 
asset portfolio consists of a treasury security, a marketable security, and a loan. We take the stochastic optimal control approach and arrive at a nonlinear second-order PDE for the value function for which it is difficult to find a solution. Our choice of asset portfolio is based on the portfolio of Gao [17]. In a pension fund setting, Gao [17] solved the optimization problem in question using the Legendre transform and dual theory. With our optimization problem similar to that in [17], we evoke the results of Gao. Based on the investment strategy that solves our optimization problem we derive a model for the Total Capital Ratio or CAR of the bank in terms of the optimal amounts of bank capital invested in the assets. Furthermore, we assume that the bank follows the optimal investment strategy while simultaneously maintaining its CAR at the fixed level of $8 \%$. We obtain a formula for the bank's asset portfolio into which is incorporated the restriction on the CAR. By means of numerical simulations we characterize the optimal investment strategy by presenting graphically the optimal proportions of capital invested in the bank's assets. In addition, we simulate and graphically illustrate the behaviours of the CAR and the modified asset portfolio subject to the optimal investment strategy. To our knowledge, a study as described above has not been done for a commercial Basel III compliant bank.

The layout of the rest of our paper is as follows. In Section 2 we present some general banking theory. More specifically, we describe the bank's assets, liabilities, and capital, all of which comprise its stylized balance sheet. We introduce the financial market in Section 3. Here we specify models for the stochastic interest rate in the market and the assets and asset portfolio of the bank. In Section 4 we formulate the optimization problem and present the optimal solution. In this section we also present the bank capital model relevant to the optimization problem. The formula for the CAR is derived in Section 5. We simulate graphically the optimal investment proportions of capital invested in the assets as well as the dynamics of the CAR. Section 6 is devoted to the derivation of the asset portfolio pertaining to a constant CAR. In this section we also provide a simulation of the modified asset portfolio obtained via the optimal investment strategy and restricted CAR. We conclude the paper with Section 7.

\section{The Commercial Banking Model}

We consider a complete and frictionless financial market which is continuously open over a fixed time interval $[0, T]$. Throughout, we assume that we are working with a probability space $(\Omega, \mathscr{F}, \mathbb{P})$, where $\mathbb{P}$ is the real world probability. We assume that the Brownian motions that appear in the dynamics of the bank items are defined on the probability space $(\Omega, \mathscr{F}, \mathbb{P})$ and that the filtration $(\mathscr{F}(t))_{t \geq 0}$ satisfies the usual conditions.

To understand the operation and management of commercial banks, we study its stylized balance sheet, which records the assets (uses of funds) and liabilities (sources of funds) of the bank.
Bank capital fulfills the role of balancing the assets and liabilities of the bank. A useful way, for our analysis, of representing the balance sheet of the bank is as follows:

$$
R(t)+S(t)+L(t)=D(t)+B(t)+C(t),
$$

where $R, S, L, D, B$, and $C$ represent the values of treasuries, securities, loans, deposits, borrowings, and capital, respectively. Each of the variables above is regarded as a function $\Omega \times \mathbb{R}_{+} \rightarrow \mathbb{R}_{+}$.

In order for a commercial bank to make a profit, it is important that the bank manages the asset side of its balance sheet properly. The latter is determined by two factors, namely, the amount of capital available to invest it has and the attitude it has toward risk and return. The bank must therefore allocate its capital optimally among its assets. Below we explain each of the items on the balance sheet of a commercial bank.

The term reserves refers to the vault cash of the bank plus the compulsory amount of its money deposited at the central bank. Reserves are used to meet the day-to-day currency withdrawals by its customers. Securities consist of treasury securities (treasuries) and marketable securities (securities). Treasuries are bonds issued by national treasuries in most countries as a means of borrowing money to meet government expenditures not covered by tax revenues. Securities, on the other hand, are stocks and bonds that can be converted to cash quickly and easily. Loans granted by the bank include business loans, mortgage loans (land loans), and consumer loans. Consumer loans include credit extended by the bank for credit card purchases. Mortgages are long term loans used to buy a house or land, where the house or land acts as collateral. Business loans are taken out by firms that borrow funds to finance their inventories, which act as collateral for the loan. A loan which has collateral (a secured loan) has a lower interest rate associated with it compared to a loan which has no collateral (unsecured loans).

Bank capital is raised by selling new equity, retaining earnings, and by issuing debt or building up loan-loss reserves. The bank's risk management department is usually responsible for calculating its capital requirements. Calculated risk capital is then approved by the bank's top executive management. Furthermore, the structure of bank capital is proposed by the Finance Department and subsequently approved by the bank's top executive management. The dynamics of bank capital is stochastic in nature because it depends in part on the uncertainty related to debt and shareholder contributions. In theory, the bank can decide on the rate at which debt and equity are raised.

Under the Basel III Accord (see [21]) the bank's capital C, also referred to as total bank capital, has the form

$$
C(t)=C_{\mathrm{T} 1}(t)+C_{\mathrm{T} 2}(t),
$$

where $C_{\mathrm{T} 1}(t)$ and $C_{\mathrm{T} 2}$ are Tier 1 and Tier 2 capital, respectively.

Tier 1 capital consists of the sum of Common Equity Tier 1 capital and Additional Tier 1 capital. Common Equity Tier 1 capital is defined as the sum of common shares issued by the bank that meet the criteria for classification as common 
shares for regulatory purposes, stock surplus resulting from the issue of instruments included in Common Equity Tier 1 capital, retained earnings, other accumulated comprehensive income and other disclosed reserves, common shares issued by consolidated subsidiaries of the bank and held by third parties that meet the criteria for inclusion in Common Equity Tier 1 capital, and regulatory adjustments applied in the calculation of Common Equity Tier 1 capital. Additional Tier 1 capital is the sum of instruments issued by the bank that meet the criteria for inclusion in Additional Tier 1 capital (and are not included in Common Equity Tier 1 capital), stock surplus resulting from the issue of instruments included in Additional Tier 1 capital, instruments issued by consolidated subsidiaries of the bank and held by third parties that meet the criteria for inclusion in Additional Tier 1 capital and are not included in Common Equity Tier 1 capital, and regulatory adjustments applied in the calculation of Additional Tier 1 capital.

Tier 2 capital is defined as the sum of the following elements: instruments issued by the bank that meet the criteria for inclusion in Tier 2 capital (and are not included in Tier 1 capital), stock surplus resulting from the issue of instruments included in Tier 2 capital, instruments issued by consolidated subsidiaries of the bank and held by third parties that meet the criteria for inclusion in Tier 2 capital and are not included in Tier 1 capital, certain loan-loss provisions, and regulatory adjustments applied in the calculation of Tier 2 capital.

Deposits refer to the money that the bank's customers place in the banking institution for safekeeping. Deposits are made to deposit accounts at a banking institution, such as savings accounts, checking accounts, and money market accounts. The account holder has the right to withdraw any deposited funds, as set forth in the terms and conditions of the account. Deposits are considered to be the main liabilities of the bank.

The term borrowings refers to the funds that commercial banks borrow from other banks and the central bank.

\section{The Financial Market Setting}

We assume that it is possible for the bank to continuously raise small levels of capital at a rate $d C(t)$. The bank is assumed to invest its capital in a market consisting of at least two types of assets (treasury and security) which can be bought and sold without incurring any transaction costs or restriction on short sales. It is assumed that the bank invests in a third asset, namely, a loan.

The first asset in the financial market is a riskless treasury. We denote its price at time $t$ by $S_{0}(t)$ and assume that its dynamics evolve according to the equation

$$
\frac{d S_{0}(t)}{S_{0}(t)}=r(t) d t, \quad S_{0}(0)=1 \text {. }
$$

The dynamics of the short rate process, $r(t)$, is given by the stochastic differential equation (SDE)

$$
d r(t)=(a-b r(t)) d t-\sigma_{r} d W_{r}(t)
$$

for $t \geq 0$ and where $\sigma_{r}=\sqrt{k_{1} r(t)+k_{2}}$. The coefficients $a, b$, $k_{1}$, and $k_{2}$, as well as the initial value $r(0)$, are all positive real constants. The above dynamics recover, as a special case, the Vasiček [22] (resp., Cox et al. [23]) dynamics when $k_{1}$ (resp., $k_{2}$ ) is equal to zero. The term structure of the interest rates is affine under the aforementioned dynamics.

The second asset in the market is a risky security whose price is denoted by $S(t), t \geq 0$. Its dynamics are given by the equation

$$
\begin{aligned}
\frac{d S(t)}{S(t)}= & r(t) d t+\sigma_{1}\left(d W_{s}(t)+\lambda_{1} d t\right) \\
& +\sigma_{2} \sigma_{r}\left(d W_{r}(t)+\lambda_{2} \sigma_{r} d t\right)
\end{aligned}
$$

with $S(0)=1$ and $\lambda_{1}, \lambda_{2}$ (resp., $\sigma_{1}, \sigma_{2}$ ) being constants (resp., positive constants) as in Deelstra et al. [24].

The third asset is a loan to be amortized over a period $[0, T]$ whose price at time $t \geq 0$ is denoted by $L(t)$. We assume that its dynamics can be described by the SDE

$$
\frac{d L(t)}{L(t)}=r(t) d t+\sigma_{L}(T-t, r(t))\left(d W_{r}(t)+\lambda_{2} \sigma_{r} d t\right) \text {. }
$$

We now model the asset portfolio of the bank. Let $X(t)$ denote the value of the asset portfolio at time $t \in[0, T]$. The dynamics of the asset portfolio are described by

$$
\begin{aligned}
d X(t)= & \theta_{r}(t) \frac{d S_{0}(t)}{S_{0}(t)}+\theta_{S}(t) \frac{d S(t)}{S(t)} \\
& +\theta_{L}(t) \frac{d L(t)}{L(t)}+d C(t),
\end{aligned}
$$

where $\theta_{S}(t), \theta_{L}(t)$, and $\theta_{r}(t)$ denote the amounts of capital invested in the two risky assets (security and loan) and in the riskless asset (treasury), respectively. Making use of the models for $S_{0}(t), S(t)$, and $L(t)$, we can rewrite (8) as

$$
\begin{aligned}
d X(t)=\{ & X(t) r(t)+\theta_{S}(t)\left[\lambda_{1} \sigma_{1}+\lambda_{2} \sigma_{2} \sigma_{r}^{2}\right] \\
& \left.+\theta_{L}(t) \lambda_{2} \sigma_{L}(T-t, r(t)) \sigma_{r}\right\} d t \\
+ & \theta_{S}(t) \sigma_{1} d W_{S}(t)+\left(\theta_{L}(t) \sigma_{L}(T-t, r(t))\right. \\
& \left.+\theta_{S}(t) \sigma_{2} \sigma_{r}\right) d W_{r}(t)+d C(t) .
\end{aligned}
$$

\section{The Portfolio Problem and Optimal Solution}

In this section we formulate the optimal control problem associated with an influx of capital at a rate

$$
d C(t)=c(t) d t, \quad C(0)>0 .
$$

We derive the HJB equation for the value function and we present the optimal solution from the methodology of Gao [17].

We wish to choose a portfolio strategy in order to maximize the expected utility of the bank's asset portfolio at a future date $T>0$. Mathematically, the stochastic optimal control problem can be stated as follows. 
Problem 1. Our objective is to maximize the expected utility of the bank's asset portfolio at a future date $T>0$. Thus we must

$$
\begin{aligned}
& \text { maximize } J\left(\theta_{S}, \theta_{L}\right)=\mathbb{E}(u(X(T))) \text {, } \\
& \text { subject to } d r(t)=(a-b r(t)) d t-\sigma_{r} d W_{r}(t) \text {, } \\
& d X(t)=\left[X(t) r(t)+\theta_{S}(t)\right. \\
& \times\left(\lambda_{1} \sigma_{1}+\lambda_{2} \sigma_{2} \sigma_{r}^{2}\right) \\
& +\theta_{L}(t) \lambda_{2} \sigma_{L}(T-t, r(t)) \sigma_{r} \\
& +c(t)] d t+\theta_{S}(t) \sigma_{1} d W_{S}(t) \\
& +\left(\theta_{L}(t) \sigma_{L}(T-t, r(t))\right. \\
& \left.+\theta_{S}(t) \sigma_{2} \sigma_{r}\right) d W_{r}(t) \\
& X(0)=x_{0}, \quad r(0)=r_{0},
\end{aligned}
$$

with $0 \leq t \leq T$, and where $X(0)=x_{0}$ and $r(0)=r_{0}$ denote the initial conditions of the optimal control problem.

In this paper we describe the bank's objective with a logarithmic utility function; that is,

$$
u(x)=\ln x, \quad \text { with } x>0 .
$$

We note that the utility function $u(\cdot)$ is strictly concave up and satisfies the Inada conditions $u^{\prime}(+\infty)=0$ and $u^{\prime}(0)=+\infty$. By using the classical tools of stochastic optimal control, we define the value function

$$
H(t, r, x)=\sup _{\theta_{S}, \theta_{L}} \mathbb{E}(u(X(T) \mid r(t)=r, X(t)=x)),
$$

$$
0<t<T
$$

The value function can be considered as a kind of utility function. The marginal utility of the value function is a constant, while the marginal utility of the original utility function $u(\cdot)$ decreases to zero as $x \rightarrow \infty$ (see Kramkov and Schachermayer [25]). The value function also inherits the convexity of the utility function (see Jonsson and Sircar [26]). Moreover, it is strictly convex for $t<T$ even if $u(\cdot)$ is not.

The maximum principle leads to the HJB equation (see Duffie [27]):

$$
\begin{aligned}
H_{t} & +a(b-r) H_{r} \\
& +\left[x r+\left(\lambda_{1} \sigma_{1}+\lambda_{2} \sigma_{2} \sigma_{r}^{2}\right) \theta_{S}+\lambda_{2} \sigma_{L} \sigma_{r} \theta_{L}+c\right] H_{x} \\
& +\frac{1}{2}\left[\sigma_{1}^{2} \theta_{S}^{2}+\left(\sigma_{L} \theta_{L}+\sigma_{2} \sigma_{r} \theta_{S}\right)^{2}\right] H_{x x}+\frac{\sigma_{r}^{2}}{2} H_{r r} \\
& -\left(\sigma_{L} \sigma_{r} \theta_{L}+\sigma_{2} \sigma_{r}^{2} \theta_{S}\right) H_{r x}=0,
\end{aligned}
$$

where we have suppressed the time variable $t$. In the HJB equation above, $H_{t}, H_{r}, H_{x}, H_{r r}, H_{x x}$, and $H_{r x}$ denote partial derivatives of first and second orders with respect to time, interest rate, and asset portfolio.
The first-order maximizing conditions for the optimal strategies $\theta_{S}$ and $\theta_{L}$ are

$$
\begin{gathered}
\theta_{S}=-\frac{\lambda_{1}}{\sigma_{1}} \frac{H_{x}}{H_{x x}}, \\
\theta_{L}=\frac{\sigma_{r}\left(\lambda_{1} \sigma_{2}-\lambda_{2} \sigma_{1}\right) H_{x}+\sigma_{1} \sigma_{r} H_{r x}}{\sigma_{1} \sigma_{L} H_{x x}} .
\end{gathered}
$$

If we put (15) into (14), we obtain a PDE for the value function $H$ :

$$
\begin{gathered}
H_{t}+a(b-r) H_{r}+\frac{\sigma_{r}^{2}}{2} H_{r r}+(x r+c) H_{x} \\
-\frac{\lambda_{1}^{2}}{2} \frac{H_{x}^{2}}{H_{x x}}-\frac{\left(\lambda_{2} \sigma_{r} H_{x}-\sigma_{r} H_{r x}\right)^{2}}{2 H_{x x}}=0 .
\end{gathered}
$$

The problem is to solve (16) for the value function $H$ and replace it in (15) in order to obtain the optimal investment strategy. The above equation is a nonlinear second-order PDE, which is very difficult to solve. The solution to this particular problem was derived in a pension fund setting by Gao [17], who did so by employing the Legendre transform and dual theory. We present the optimal solution from Gao [17] in Remark 2 below.

At this point we shall specify a particular candidate for the function $\sigma_{L}$ appearing in (7). We assume $\sigma_{L}$ to take the form

$$
\sigma_{L}(T-t, r(t))=h(T-t) \sigma_{r}
$$

with

$$
\begin{gathered}
h(t)=\frac{2\left(e^{m t}-1\right)}{m-\left(b-k_{1} \lambda_{2}\right)+e^{m t}\left(m+b-k_{1} \lambda_{2}\right)}, \\
m=\sqrt{\left(b-k_{1} \lambda_{2}\right)^{2}+2 k_{1}} .
\end{gathered}
$$

Remark 2. The solution of Problem 1 is obtained by solving (16) in terms of $H$ and substituting the result into (15). The optimal solution is as follows.

The optimal amount of bank capital invested in the loan is

$$
\begin{aligned}
\theta_{L}=\frac{\sigma_{r}\left(\lambda_{2} \sigma_{1}-\lambda_{1} \sigma_{2}\right) x}{\sigma_{1} \sigma_{L}} & \\
-\sigma_{r} c(t)[ & {\left[\frac{\left(\lambda_{1} \sigma_{2}-\lambda_{2} \sigma_{1}\right) \bar{a}_{\overline{T-t} \mid r_{t}}}{\sigma_{1} \sigma_{L}}\right.} \\
& \left.+\frac{\bar{a}_{\overline{T-t \mid} r_{t}}-(T-t)\left(1-r \bar{a}_{\overline{T-t} \mid r_{t}}\right)}{r \sigma_{L}}\right],
\end{aligned}
$$


or if we denote the optimal proportion of capital invested in the loan by $\eta_{L}$, then we can write

$$
\begin{aligned}
& \eta_{L}= \frac{\sigma_{r}\left(\lambda_{2} \sigma_{1}-\lambda_{1} \sigma_{2}\right)}{\sigma_{1} \sigma_{L}} \\
&-\frac{\sigma_{r} c(t)}{x}\left[\frac{\left(\lambda_{1} \sigma_{2}-\lambda_{2} \sigma_{1}\right) \bar{a}_{\overline{T-t} \mid r_{t}}}{\sigma_{1} \sigma_{L}}\right. \\
&\left.+\frac{\bar{a}_{\overline{T-t} \mid r_{t}}-(T-t)\left(1-r \bar{a}_{\overline{T-t} \mid r_{t}}\right)}{r \sigma_{L}}\right] .
\end{aligned}
$$

Furthermore, the optimal amount of capital invested in the security is given by

$$
\theta_{S}=\frac{\lambda_{1}}{\sigma_{1}}\left(x+c(t) \bar{a}_{\overline{T-t} \mid r_{t}}\right)
$$

or if $\eta_{S}$ denotes the optimal proportion of capital invested in the security, then

$$
\eta_{S}=\frac{\lambda_{1}}{\sigma_{1}}+\frac{c(t) \bar{a}_{\overline{T-t} \mid r_{t}}}{x} .
$$

According to the above models, we may write the optimal amount of capital invested in the treasury as

$$
\theta_{r}=x-\theta_{L}-\theta_{S}
$$

and hence

$$
\eta_{r}=1-\eta_{L}-\eta_{S}
$$

where $\eta_{r}$ is the optimal proportion of capital invested in the treasury. The expression $\bar{a} \frac{}{T-t}$ denotes a continuous annuity of duration $T-t$.

\section{The Total Capital Ratio}

We now proceed to derive the dynamics of the Total Capital Ratio (CAR). In order to do so, we first derive the dynamics of the TRWAs of the bank. In addition, we simulate the optimal proportions of bank capital invested in the assets and the CAR at the end of this section. The dynamics of the TRWAs and CAR are, respectively, derived in the remark and proposition below. We still assume that the bank capital evolves according to $(10)$.

Remark 3. Suppose that, at time $t$, the TRWAs can be described by the SDE

$$
\begin{aligned}
d a_{\mathrm{rw}}(t)= & 0 \times \theta_{r}(t) \frac{d S_{0}(t)}{S_{0}(t)}+0.2 \times \theta_{S}(t) \frac{d S(t)}{S(t)} \\
& +0.5 \times \theta_{L}(t) \frac{d L(t)}{L(t)}+c(t) d t,
\end{aligned}
$$

where $0,0.2$, and 0.5 are the risk-weights associated with, respectively, the treasury, security, and loan under the Basel
III Accord. By simplifying (25), the expression for $d a_{\mathrm{rW}}(t)$ reduces to the following:

$$
\begin{aligned}
d a_{\mathrm{rW}}( & t) \\
= & {\left[0.2 \theta_{S}(t)\left(r(t)+\sigma_{1} \lambda_{1}+\sigma_{2} \sigma_{r}^{2} \lambda_{2}\right)\right.} \\
& \left.+0.5 \theta_{L}(t)\left(r(t)+\sigma_{L}(T-t, r(t)) \lambda_{2} \sigma_{r}\right)+c(t)\right] d t \\
+ & 0.2 \theta_{S}(t) \sigma_{1} d W_{S}(t) \\
+ & {\left[0.2 \theta_{S}(t) \sigma_{2} \sigma_{r}\right.} \\
& \left.+0.5 \theta_{L}(t) \sigma_{L}(T-t, r(t))\right] d W_{r}(t) .
\end{aligned}
$$

In the proof of the proposition below we make use of Itô's Lemma and Itô's Product Rule, for which we refer to the book [28] by Øksendal.

Proposition 4. With the dynamics of the total bank capital, $C(t)$, given by the ODE in (10), and with the dynamics of the TRWAs, $a_{r w}$, given by (26), we can write the dynamics of the Total Capital Ratio or CAR at time t as

$$
\begin{aligned}
d \Lambda(t)=\Psi_{1} d t+\frac{C(t)}{a_{r w}^{2}(t)}[ & \left(-\Psi_{2}+\frac{\Psi_{3}}{a_{r w}(t)}\right) d t \\
& \left.-\left(\Xi_{1} d W_{S}(t)+\Xi_{2} d W_{r}(t)\right)\right],
\end{aligned}
$$

where

$$
\begin{gathered}
\Psi_{1}=\frac{c(t)}{a_{r w}(t)}, \\
\Psi_{2}=0.2 \theta_{S}(t)\left(r(t)+\sigma_{1} \lambda_{1}+\sigma_{2} \sigma_{r}^{2} \lambda_{2}\right) \\
+0.5 \theta_{L}(t)\left(r(t)+\sigma_{L}(T-t, r(t)) \lambda_{2} \sigma_{r}\right)+c(t), \\
\Psi_{3}=\left(0.2 \theta_{S}(t) \sigma_{1}\right)^{2}+\left(0.2 \theta_{S}(t) \sigma_{2} \sigma_{r}\right. \\
\left.+0.5 \theta_{L}(t) \sigma_{L}(T-t, r(t))\right)^{2}, \\
\Xi_{1}=0.2 \theta_{S}(t) \sigma_{1}, \\
\Xi_{2}=0.2 \theta_{S}(t) \sigma_{2} \sigma_{r}+0.5 \theta_{L}(t) \sigma_{L}(T-t, r(t)) .
\end{gathered}
$$

Proof. We mainly use Itô's general formula to derive (27). Let $\Phi\left(a_{\mathrm{rW}}(t)\right)=1 /\left(a_{\mathrm{rW}}(t)\right)$. Then by Itô's Lemma,

$$
\begin{aligned}
d \Phi & \left(a_{\mathrm{rW}}(t)\right) \\
= & \dot{\Phi}\left(a_{\mathrm{rw}}(t)\right) d t+\Phi^{\prime}\left(a_{\mathrm{rW}}(t)\right) d a_{\mathrm{rw}}(t) \\
& +\frac{1}{2} \Phi^{\prime \prime}\left(a_{\mathrm{rw}}(t)\right)\left[d a_{\mathrm{rw}}(t)\right]^{2} \\
= & 0 d t-\frac{d a_{\mathrm{rw}}(t)}{a_{\mathrm{rW}}^{2}(t)}+\frac{\left[d a_{\mathrm{rW}}(t)\right]^{2}}{a_{\mathrm{rW}}^{3}(t)}
\end{aligned}
$$




$$
\begin{gathered}
=\left\{-\frac{1}{a_{\mathrm{rW}}^{2}(t)}\left[0.2 \theta_{S}(t)\left(r(t)+\sigma_{1} \lambda_{1}+\sigma_{2} \sigma_{r}^{2} \lambda_{2}\right)\right.\right. \\
+0.5 \theta_{L}(t)\left(r(t)+\sigma_{L}(T-t, r(t)) \lambda_{2} \sigma_{r}\right) \\
+c(t)]+\frac{1}{a_{\mathrm{rW}}^{3}(t)}\left[\left(0.2 \theta_{S}(t) \sigma_{1}\right)^{2}\right. \\
+\left(0.2 \theta_{S}(t) \sigma_{2} \sigma_{r}\right. \\
+0.5 \theta_{L}(t) \sigma_{L} \\
\left.\left.\quad \times(T-t, r(t)))^{2}\right]\right\} d t \\
-\frac{1}{a_{\mathrm{rW}}^{2}(t)}\left[0.2 \theta_{S}(t) \sigma_{1} d W_{S}(t)\right. \\
+\left(0.2 \theta_{S}(t) \sigma_{2} \sigma_{r}\right. \\
\left.\left.+0.5 \theta_{L}(t) \sigma_{L}(T-t, r(t))\right) d W_{r}(t)\right] .
\end{gathered}
$$

Let $\Lambda(t)$ denote the CAR at time $t$ for $t \in[0, T]$. Then by definition (see (1)), we can write $\Lambda(t)$ as

$$
\Lambda(t)=\frac{C(t)}{a_{\mathrm{rW}}(t)}=C(t) \Phi\left(a_{\mathrm{rW}}(t)\right) .
$$

We apply Itô's Product Rule to $\Lambda(t)=C(t) \Phi\left(a_{\mathrm{rw}}(t)\right)$ to find an expression for $d \Lambda(t)$ :

$d \Lambda(t)$

$$
\begin{gathered}
=d C(t) \Phi\left(a_{\mathrm{rW}}(t)\right)+C(t) d \Phi\left(a_{\mathrm{rW}}(t)\right) \\
=\frac{c(t)}{a_{\mathrm{rW}}(t)} d t+C(t) d \Phi\left(a_{\mathrm{rW}}(t)\right) \\
=\frac{c(t)}{a_{\mathrm{rW}}(t)} d t \\
+C(t)\left\{\left\{-\frac{1}{a_{\mathrm{rW}}^{2}(t)}\left[0.2 \theta_{S}(t)\left(r(t)+\sigma_{1} \lambda_{1}+\sigma_{2} \sigma_{r}^{2} \lambda_{2}\right)\right.\right.\right. \\
+0.5 \theta_{L}(t)\left(r(t)+\sigma_{L}(T-t, r(t))\right. \\
\left.\left.\times \lambda_{2} \sigma_{r}\right)+c(t)\right]+\frac{1}{a_{\mathrm{rW}}^{3}(t)} \\
\times\left[\left(0.2 \theta_{S}(t) \sigma_{1}\right)^{2}+\left(0.2 \theta_{S}(t) \sigma_{2} \sigma_{r}+0.5 \theta_{L}\right.\right. \\
\left.\left.\left.\times(t) \sigma_{L}(T-t, r(t))\right)^{2}\right]\right\} d t \\
\quad \frac{1}{a_{\mathrm{rW}}^{2}(t)}\left[0.2 \theta_{S}(t) \sigma_{1} d W_{S}(t)\right. \\
+\left(0.2 \theta_{S}(t) \sigma_{2} \sigma_{r}+0.5 \theta_{L}(t) \sigma_{L}\right. \\
\left.\left.\times(T-t, r(t))) d W_{r}(t)\right]\right\}
\end{gathered}
$$

$$
\begin{aligned}
=\Psi_{1} d t+C(t)[ & \left(-\frac{1}{a_{\mathrm{rW}}^{2}(t)} \Psi_{2}+\frac{1}{a_{\mathrm{rW}}^{3}(t)} \Psi_{3}\right) d t-\frac{1}{a_{\mathrm{rW}}^{2}(t)} \\
\times & \left.\left(\Xi_{1} d W_{S}(t)+\Xi_{2} d W_{r}(t)\right)\right] \\
=\Psi_{1} d t+\frac{C(t)}{a_{\mathrm{rW}}^{2}(t)}[ & \left(-\Psi_{2}+\frac{\Psi_{3}}{a_{\mathrm{rW}}(t)}\right) d t \\
& \left.-\left(\Xi_{1} d W_{S}(t)+\Xi_{2} d W_{r}(t)\right)\right],
\end{aligned}
$$

where we have defined

$$
\begin{gathered}
\Psi_{1}=\frac{c(t)}{a_{\mathrm{rw}}(t)}, \\
\Psi_{2}=0.2 \theta_{S}(t)\left(r(t)+\sigma_{1} \lambda_{1}+\sigma_{2} \sigma_{r}^{2} \lambda_{2}\right) \\
+0.5 \theta_{L}(t)\left(r(t)+\sigma_{L}(T-t, r(t)) \lambda_{2} \sigma_{r}\right)+c(t), \\
\Psi_{3}=\left(0.2 \theta_{S}(t) \sigma_{1}\right)^{2} \\
+\left(0.2 \theta_{S}(t) \sigma_{2} \sigma_{r}+0.5 \theta_{L}(t) \sigma_{L}(T-t, r(t))\right)^{2}, \\
\Xi_{1}=0.2 \theta_{S}(t) \sigma_{1}, \\
\Xi_{2}=0.2 \theta_{S}(t) \sigma_{2} \sigma_{r}+0.5 \theta_{L}(t) \sigma_{L}(T-t, r(t)) .
\end{gathered}
$$

This concludes the proof.

We provide a numerical simulation in order to characterize the behaviour of the CAR, $\Lambda$. We assume that the interest rate follows the CIR dynamics $\left(k_{2}=0\right)$ and that the financial market consists of a treasury, a security, and a loan. Furthermore, we consider an investment horizon of $T=10$ years and that capital is raised at the fixed rate of $c=0.13$. The rest of the parameters of the simulation are

$$
\begin{gathered}
a=0.0118712, \quad b=0.0339, \\
k_{1}=0.00118712, \quad \sigma_{1}=0.1475, \\
\lambda_{1}=0.045, \quad \sigma_{2}=0.295, \quad \lambda_{2}=0.09
\end{gathered}
$$

with initial conditions

$$
C(0)=1, \quad r(0)=0.075,
$$

$$
X(0)=1.5, \quad a_{\mathrm{rW}}(0)=1.4, \quad \Lambda(0)=0.08 .
$$

In Figure 1 we characterize the optimal investment strategy that solves Problem 1 by simulating the optimal proportions of capital invested in the assets. The optimal investment strategy depicted in Figure 1 leads to the CAR in Figure 4. By diversifying its asset portfolio according to the optimal strategy illustrated by Figure 1, we note that the bank maintains its CAR in such a manner that it is above the minimum required level of $8 \%$. Since the bank complies with the minimum required ratio of total capital to TRWAs 


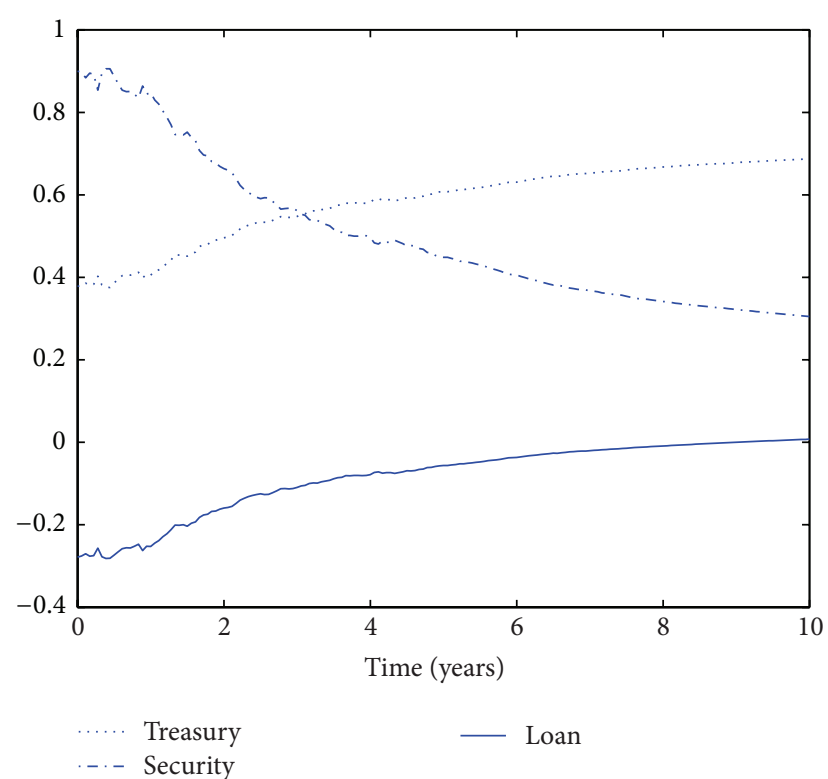

FIGURE 1: A simulation of the optimal proportions $\eta_{r}(t), \eta_{S}(t)$, and $\eta_{L}(t)$ of bank capital invested in the assets, given a constant stream of capital inflow.

as imposed by the Basel III Accord, it is guaranteed the ability to absorb reasonable levels of losses before becoming insolvent over the 10-year period. We also simulate the optimal asset portfolio and TRWAs in Figures 2 and 3, respectively. An interesting observation is that the TRWAs attain its maximum at the end of the investment period under consideration. This is due to the optimal amounts of bank capital, $\theta_{S}$ and $\theta_{L}$, invested, respectively, in the security and loan being embedded in the formula for the TRWAs in (25). The volatile natures of the quantities simulated above are consistent with the stochastic variables used throughout the paper.

\section{The Asset Portfolio for a Constant CAR}

We now set out to modify the asset portfolio of Problem 1 in such a way as to maintain the Total Capital Ratio or CAR at a constant rate of $8 \%$. To this end we need to have the bank capital $C(t)$ to be stochastic. We assume that the stochastic term is sufficiently small in order to use the solution of Problem 1 as a reasonable approximation. The actual form of $C(t)$ is deduced from the identity $C(t)=0.08 a_{\mathrm{rw}}$. The formula for the asset portfolio is given in Remark 5 below.

Remark 5. At time $t$ the dynamics of the asset portfolio, $Y(t)$, of the bank investing its capital according to the optimal investment strategy from Problem 1 and, in addition, maintaining its CAR at $8 \%$, can be written as

$$
d Y(t)=\chi_{1} d t+\chi_{2} d W_{S}(t)+\chi_{3} d W_{r}(t),
$$

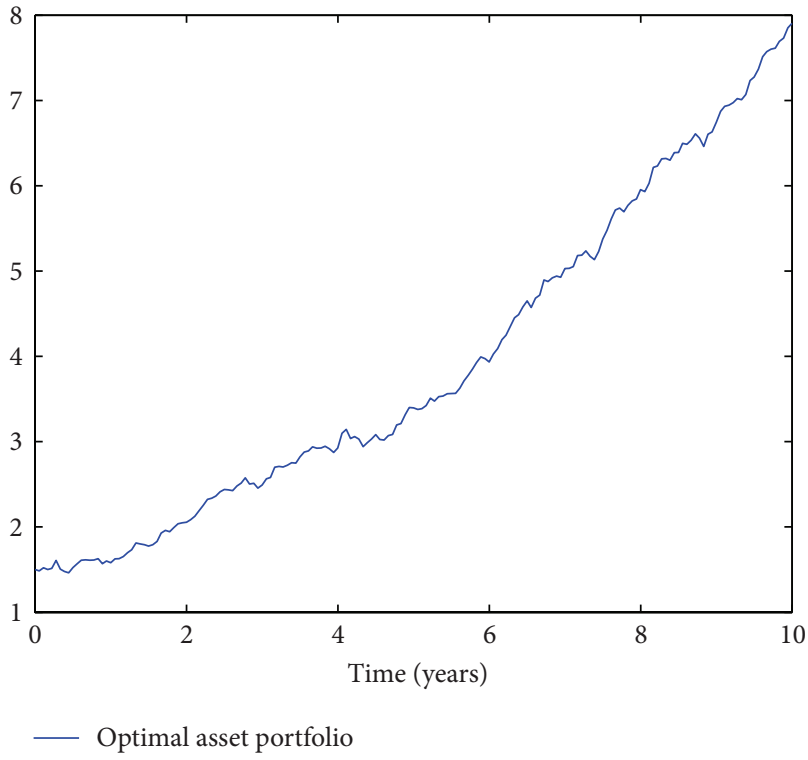

FIGURE 2: A simulation of $X(t)$, the optimal asset portfolio, given a constant stream of capital inflow.

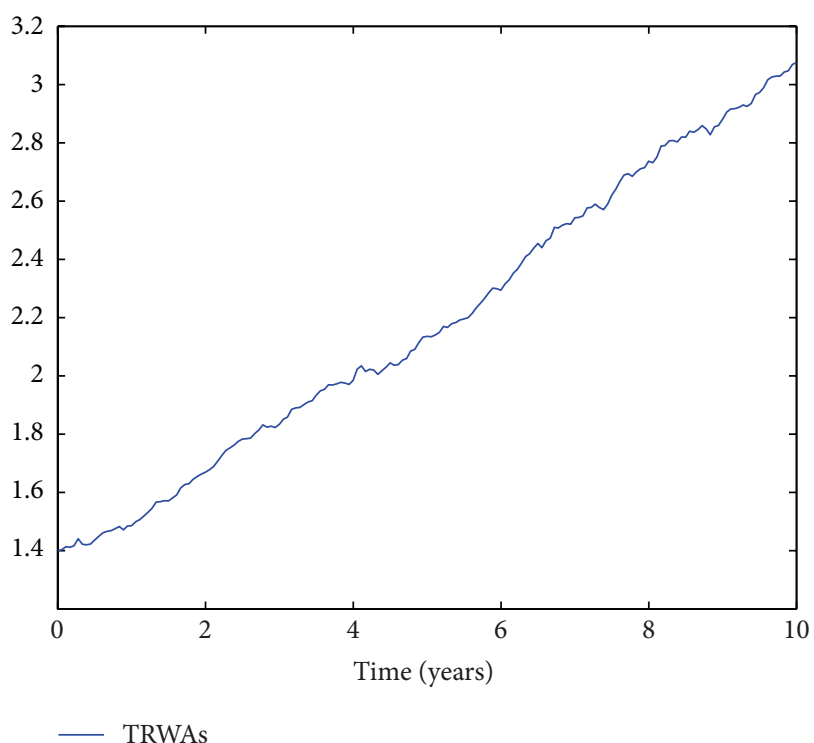

FIGURE 3: A simulation of the total risk-weighted assets, $a_{\mathrm{rw}}(t)$, given a constant stream of capital inflow.

where

$$
\begin{aligned}
& \chi_{1}=\left(X(t)-\theta_{L}(t)-\theta_{S}(t)\right) r(t) \\
&+\frac{117}{115} \theta_{S}(t)\left(r(t)+\sigma_{1} \lambda_{1}+\sigma_{2} \sigma_{r}^{2} \lambda_{2}\right) \\
&+\frac{24}{23} \theta_{L}(t)\left(r(t)+\sigma_{L}(T-t, r(t)) \lambda_{2} \sigma_{r}\right), \\
& \chi_{2}=\frac{117}{115} \theta_{S}(t) \sigma_{1}, \\
& \chi_{3}=\frac{117}{115} \theta_{S}(t) \sigma_{2} \sigma_{r}+\frac{24}{23} \theta_{L}(t) \sigma_{L}(T-t, r(t)) .
\end{aligned}
$$




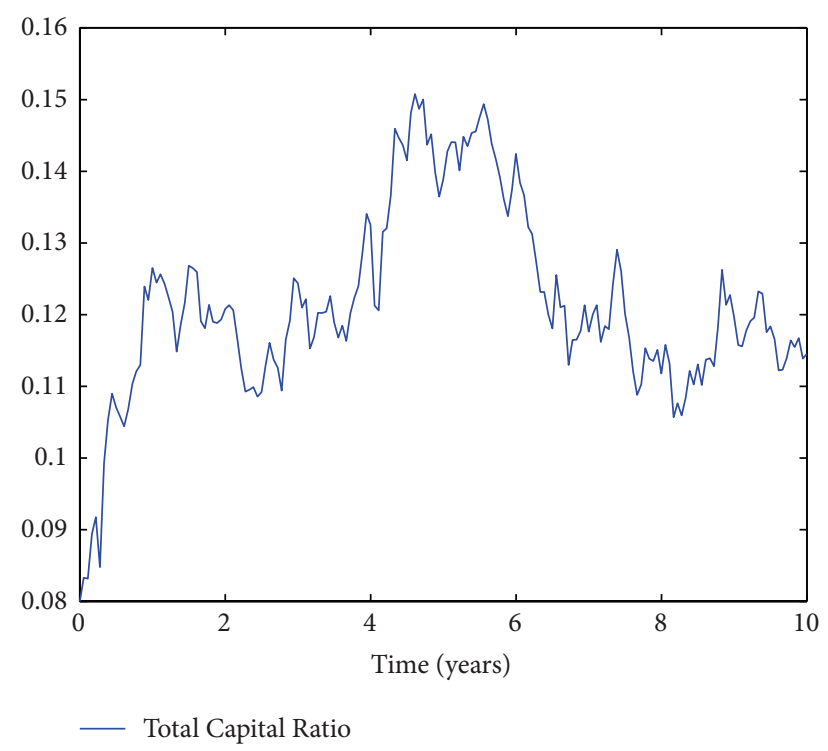

Figure 4: A simulation of $\Lambda(t)$, the Total Capital Ratio, given a constant stream of capital inflow.

The argument for this remark goes as follows. From the condition $\Lambda(t)=C(t) / a_{\mathrm{rw}}(t)=0.08$, we have $d C(t) / 0.08=$ $d a_{\mathrm{rw}}(t)$. By substituting $d C(t) / 0.08$ for the left hand side of (25) and replacing the $c(t) d t$ of (25) by $d C(t)$, we obtain

$$
\left(\frac{1}{0.08}-1\right) d C(t)=0.2 \theta_{S}(t) \frac{d S(t)}{S(t)}+0.5 \theta_{L}(t) \frac{d L(t)}{L(t)} .
$$

Solving $d C(t)$ in terms of $\theta_{S}(t)(d S(t) / S(t))$ and $\theta_{L}(t)(d L(t) /$ $L(t))$ yields

$$
d C(t)=\frac{2}{115} \theta_{S}(t) \frac{d S(t)}{S(t)}+\frac{1}{23} \theta_{L}(t) \frac{d L(t)}{L(t)} .
$$

Substituting the above expression as the $d C(t)$ of (8) gives

$$
\begin{aligned}
d Y(t)= & \theta_{r}(t) \frac{d S_{0}(t)}{S_{0}(t)}+\frac{117}{115} \theta_{S}(t) \frac{d S(t)}{S(t)} \\
& +\frac{24}{23} \theta_{L}(t) \frac{d L(t)}{L(t)} .
\end{aligned}
$$

By simplifying this expression in terms of the models of $d S_{0}(t) / S_{0}(t), d S(t) / S(t)$, and $d L(t) / L(t)$, and by recalling that $\theta_{r}(t)=X(t)-\theta_{L}(t)-\theta_{S}(t)$, we can eventually write $d Y(t)$ as

$$
\begin{aligned}
d Y(t)=[ & \left(X(t)-\theta_{L}(t)-\theta_{S}(t)\right) r(t) \\
& +\frac{117}{115} \theta_{S}(t)\left(r(t)+\sigma_{1} \lambda_{1}+\sigma_{2} \sigma_{r}^{2} \lambda_{2}\right) \\
& \left.+\frac{24}{23} \theta_{L}(t)\left(r(t)+\sigma_{L}(T-t, r(t)) \lambda_{2} \sigma_{r}\right)\right] d t
\end{aligned}
$$

$$
\begin{aligned}
+\frac{117}{115} \theta_{S}(t) \sigma_{1} d W_{S}+ & {\left[\frac{117}{115} \theta_{S}(t) \sigma_{2} \sigma_{r}+\frac{24}{23} \theta_{L}(t) \sigma_{L}\right.} \\
& \times(T-t, r(t))] d W_{r}
\end{aligned}
$$

By writing the coefficients of $d t, d W_{S}(t)$, and $d W_{r}(t)$ of the above expression, respectively, as $\chi_{1}, \chi_{2}$, and $\chi_{3}$, the asserted expression for $d Y(t)$ emerges.

We now characterize the behaviour of the modified asset portfolio given by (35). We consider an interest rate following the CIR dynamics $\left(k_{2}=0\right)$ and an investment horizon of $T=$ 10 years. A capital contribution rate of $c=0$ is considered, while the rest of the simulation parameters are

$$
\begin{gathered}
a=0.0118712, \quad b=0.0339, \\
k_{1}=0.00118712, \quad \sigma_{1}=0.1475, \\
\lambda_{1}=0.045, \quad \sigma_{2}=0.295, \quad \lambda_{2}=0.09
\end{gathered}
$$

with initial conditions

$$
\begin{aligned}
& C(0)=1, \quad r(0)=0.075, \\
& X(0)=1.5, \quad Y(0)=1.5 .
\end{aligned}
$$

For a bank that diversifies its asset portfolio according to the solution of Problem 1 and maintains its CAR at a constant value of $8 \%$, we simulate the modified version of its asset portfolio in Figure 5. In this simulation we control the bank capital $C(t)$ as in Figure 6 during the 10-year investment period. We note that the growth of the modified asset portfolio given by (35) is much slower compared to the original asset portfolio of Problem 1. For the specific simulation shown the modified asset portfolio is maximal at time $T=10$ years.

\section{Conclusion}

In this paper we consider asset portfolio and capital adequacy management in banking. In particular, our objective is to maximize an expected utility of a Basel III compliant commercial bank's asset portfolio at a future date $T>0$. From the optimal investment strategy that solves our investment problem we derive a dynamic formula for the Total Capital Ratio or Capital Adequacy Ratio (CAR). In addition, we derive a modified version of the bank's asset portfolio that would be obtained should the bank simultaneously follow the optimal investment strategy and maintain its CAR at a constant level of $8 \%$. This modified portfolio is extremely important since it takes maximum advantage by keeping the Total Capital Ratio exactly at the threshold. This means that all the funding available for investment is indeed being invested. 


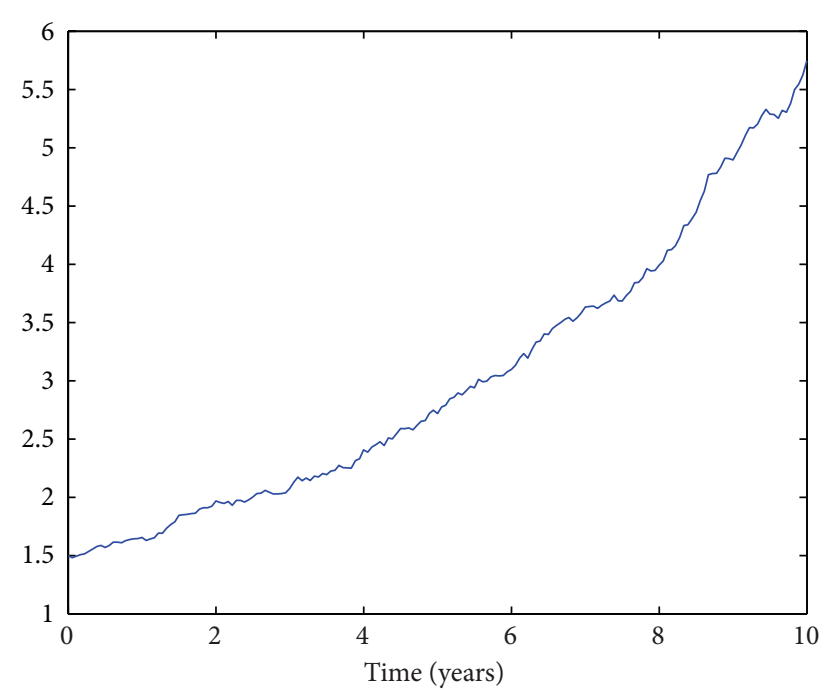

_ Modified asset portfolio

Figure 5: A simulation of $Y(t)$, the modified asset portfolio required to maintain the Total Capital Ratio at $8 \%$.

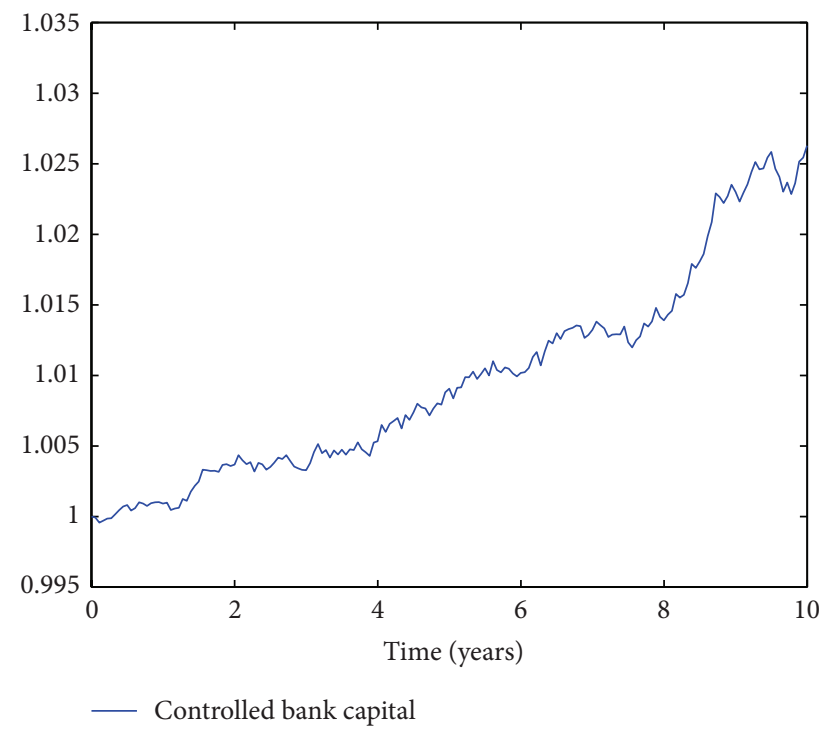

FIGURE 6: A simulation of the bank capital, $C(t)$, required to maintain the Total Capital Ratio at $8 \%$.

We present a simulation study in which we illustrate the optimal investment strategy and characterize the corresponding CAR and modified asset portfolio. The main results of our study are as follows. The optimal investment strategy for the bank is to diversify its asset portfolio away from the risky assets, that is, the security and loan and towards the riskless treasury. This is in accordance with the banking literature on optimal asset management in which it is reported that banks diversify their asset portfolios in an attempt to lower risk (see Dangl and Lehar [29] and Decamps et al. [30] for instance). In doing so, the bank maintains its CAR above the Basel III prescribed minimum level of $8 \%$. Of course, in line with Basel III standards, the bank is able to absorb reasonable levels of unexpected losses before becoming insolvent. Furthermore, in order to derive the asset portfolio at constant (minimum) CAR value, the level of bank capital needs to be controlled. This is done modifying the deterministic capital model to be stochastic. We find that the asset portfolio at constant (minimum) CAR value grows considerably slower than the asset portfolio of the original investment problem, but that this modified portfolio is still maximal at the end of the investment period under consideration. An interesting observation is that our bank raises capital while at the same time lowering risk. A possible explanation for this is that the bank holds low capital Conservation Buffers, which it is trying to rebuild (see Heid et al. [31]). In contrast, banks with large buffers maintain their capital buffer by increasing risk when capital increases [31].

In future research it would be interesting to go beyond the simulated data approach and model the CAR and bank asset portfolio at constant (minimum) CAR value using real data sourced from, for example, the US Federal Deposit Insurance Corporation (FDIC).

\section{Conflict of Interests}

The authors declare that there is no conflict of interests regarding the publication of this paper.

\section{Acknowledgment}

The authors acknowledge financial support from the National Research Foundation of South Africa.

\section{References}

[1] Basel Committee on Banking Supervision, International Convergence of Capital Measurements and Capital Standards: A Revised Framework, Bank for International Settlements, 2004.

[2] A. V. Thakor, "Capital requirements, monetary policy, and aggregate bank lending: theory and empirical evidence," Journal of Finance, vol. 51, no. 1, pp. 279-324, 1996.

[3] E.-L. von Thadden, "Bank capital adequacy regulation under the new Basel accord," Journal of Financial Intermediation, vol. 13, no. 2, pp. 90-95, 2004.

[4] C. H. Fouche, J. Mukuddem-Petersen, and M. A. Petersen, "Continuous-time stochastic modelling of capital adequacy ratios for banks," Applied Stochastic Models in Business and Industry, vol. 22, no. 1, pp. 41-71, 2006.

[5] J. Mukuddem-Petersen and M. A. Petersen, "Optimizing asset and capital adequacy management in banking," Journal of Optimization Theory and Applications, vol. 137, no. 1, pp. 205$230,2008$.

[6] M. P. Mulaudzi, M. A. Petersen, and I. M. Schoeman, "Optimal allocation between bank loans and treasuries with regret," Optimization Letters, vol. 2, no. 4, pp. 555-566, 2008.

[7] M. Jayadev, "Basel III implementation: issues and challenges for Indian banks," IIMB Management Review, vol. 25, pp. 115-130, 2013.

[8] M. A. Petersen and J. Mukuddem-Petersen, Basel III Liquidity Regulation and Its Implications, Business Expert Press, McGrawHill, New York, NY, USA, 2013. 
[9] R. Merton, "Lifetime portfolio selection under uncertainty: the continuous-time case," Review of Economics and Statistics, vol. 51, pp. 247-257, 1969.

[10] R. C. Merton, "Optimum consumption and portfolio rules in a continuous-time model," Journal of Economic Theory, vol. 3, no. 4, pp. 373-413, 1971.

[11] J. Mukuddem-Petersen and M. A. Petersen, "Bank management via stochastic optimal control," Automatica, vol. 42, no. 8, pp. 1395-1406, 2006.

[12] J. Mukuddem-Petersen, M. A. Petersen, I. M. Schoeman, and B. A. Tau, "Maximizing banking profit on a random time interval," Journal of Applied Mathematics, vol. 2007, Article ID 29343, 22 pages, 2007.

[13] J. Mukuddem-Petersen, M. P. Mulaudzi, M. A. Petersen, and I. M. Schoeman, "Optimal mortgage loan securitization and the subprime crisis," Optimization Letters, vol. 4, no. 1, pp. 97-115, 2010.

[14] F. Gideon, M. A. Petersen, J. Mukuddem-Petersen, and L. N. P. Hlatshwayo, "Basel III and the net stable funding ratio," ISRN Applied Mathematics, vol. 2013, Article ID 582707, 20 pages, 2013.

[15] M. A. Petersen, M. C. Senosi, and J. Mukuddem-Petersen, Subprime Mortgage Models, Nova, New York, NY, USA, 2011.

[16] J. Xiao, Z. Hong, and C. Qin, "The constant elasticity of variance (CEV) model and the Legendre transform-dual solution for annuity contracts," Insurance, vol. 40, no. 2, pp. 302-310, 2007.

[17] J. Gao, "Stochastic optimal control of DC pension funds," Insurance, vol. 42, no. 3, pp. 1159-1164, 2008.

[18] J. Gao, "Optimal investment strategy for annuity contracts under the constant elasticity of variance (CEV) model," Insurance, vol. 45, no. 1, pp. 9-18, 2009.

[19] J. Gao, "An extended CEV model and the Legendre transformdual-asymptotic solutions for annuity contracts," Insurance, vol. 46, no. 3, pp. 511-530, 2010.

[20] E. J. Jung and J. H. Kim, "Optimal investment strategies for the HARA utility under the constant elasticity of variance model," Insurance, vol. 51, no. 3, pp. 667-673, 2012.

[21] Basel Committee on Banking Supervision, Basel III: A Global Regulating Framework for More Resilient Banks and Banking Systems, 2010.

[22] O. E. Vasiček, "An equilibrium characterization of the term structure," Journal of Finance Economics, vol. 5, pp. 177-188, 1977.

[23] J. C. Cox, J. E. Ingersoll, Jr., and S. A. Ross, "A theory of the term structure of interest rates," Econometrica, vol. 53, no. 2, pp. 385407, 1985.

[24] G. Deelstra, M. Grasselli, and P.-F. Koehl, "Optimal investment strategies in the presence of a minimum guarantee," Insurance, vol. 33, no. 1, pp. 189-207, 2003.

[25] D. Kramkov and W. Schachermayer, "The asymptotic elasticity of utility functions and optimal investment in incomplete markets," The Annals of Applied Probability, vol. 9, no. 3, pp. 904-950, 1999.

[26] M. Jonsson and R. Sircar, "Optimal investment problems and volatility homogenization approximations," in Modern Methods in Scientific Computing and Applications, vol. 75 of NATO Science Series, pp. 255-281, Kluwer Academic, Dodrecht, The Netherlands, 2002.

[27] D. Duffie, Dynamic Asset Pricing Theory, Princeton University Press, 3rd edition, 2001.

[28] B. Øksendal, Stochastic Differential Equations: An Introduction With Applications, Springer, New York, NY, USA, 2000.
[29] T. Dangl and A. Lehar, "Value-at-risk vs. building block regulation in banking," Journal of Financial Intermediation, vol. 13, no. 2, pp. 96-131, 2004.

[30] J.-P. Decamps, J.-C. Rochet, and B. Roger, "The three pillars of Basel II: optimizing the mix," Journal of Financial Intermediation, vol. 13, no. 2, pp. 132-155, 2004.

[31] F. Heid, D. Porath, and S. Stolz, Does Capital Regulation Matter for Bank Behaviour? Evidence for German Savings Banks, vol. 3 of Discussion Paper Series 2: Banking and Financial Studies, Deutsche Bundesbank, Research Centre, 2004. 


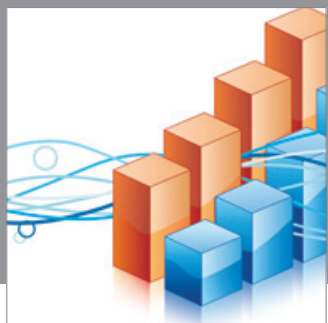

Advances in

Operations Research

mansans

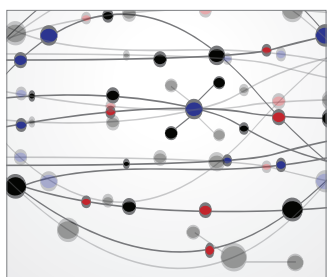

The Scientific World Journal
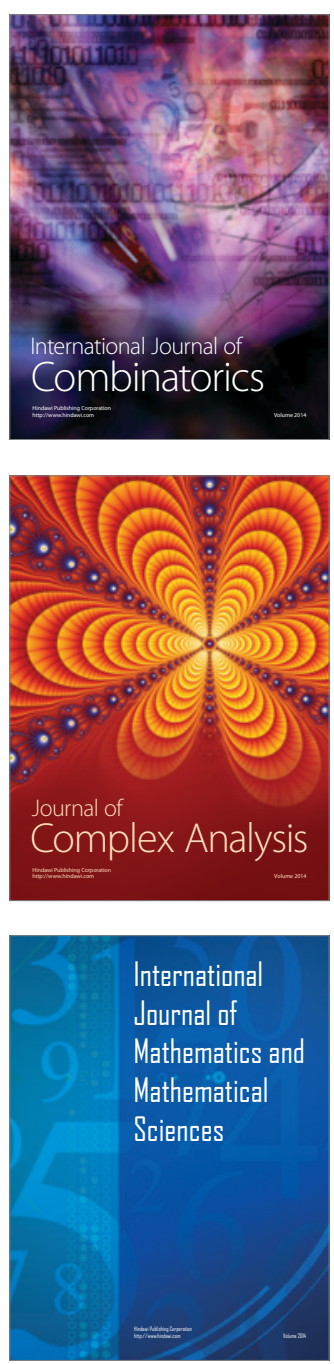
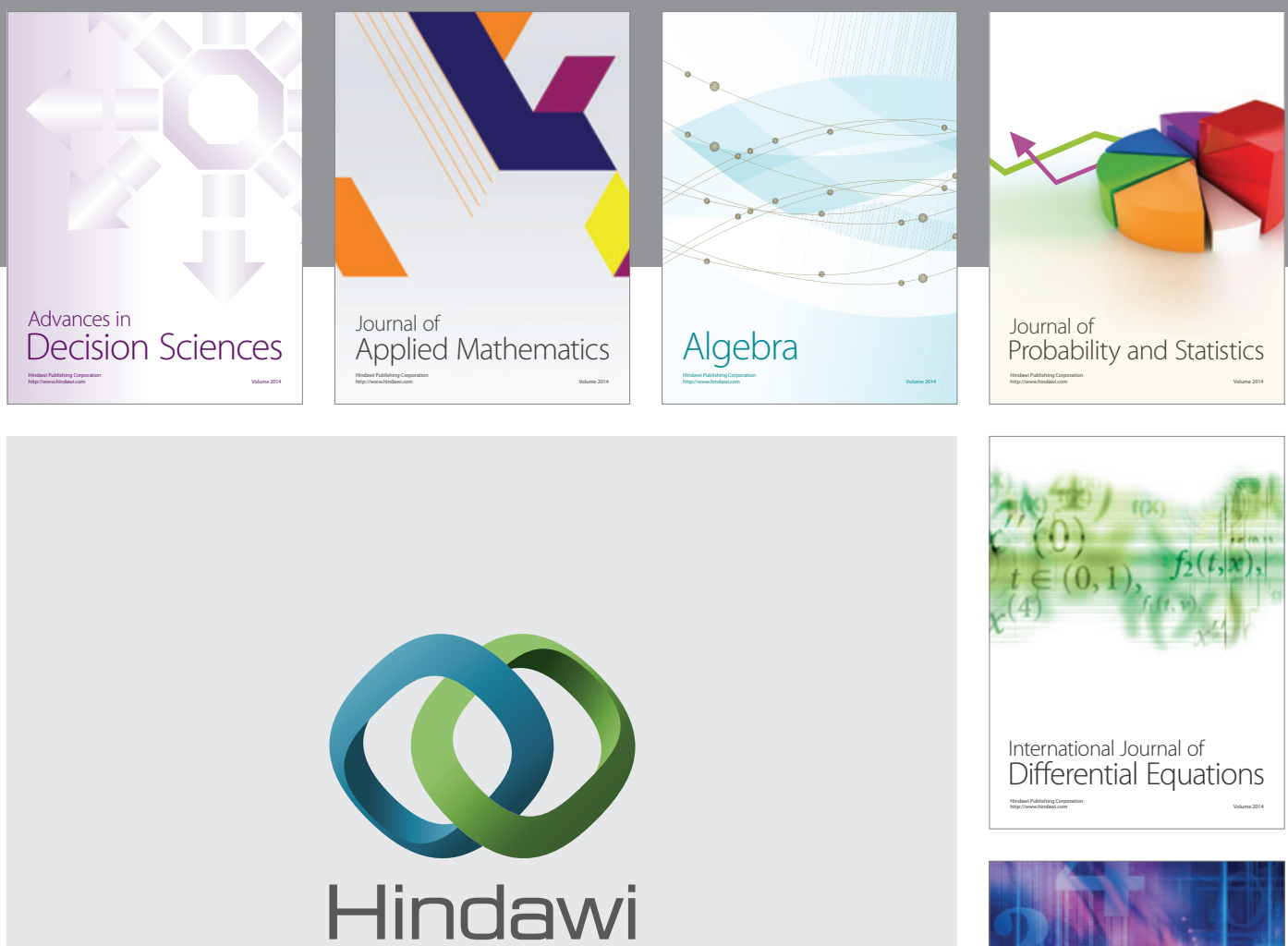

Submit your manuscripts at http://www.hindawi.com
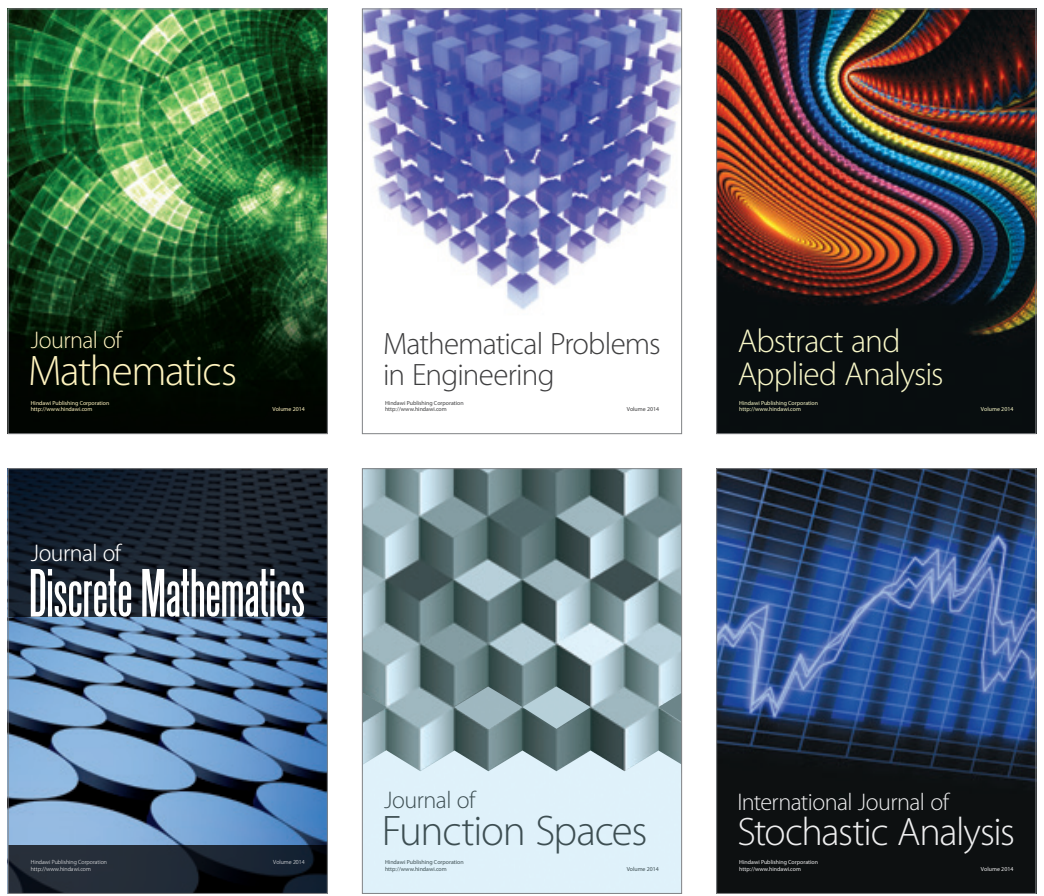

Journal of

Function Spaces

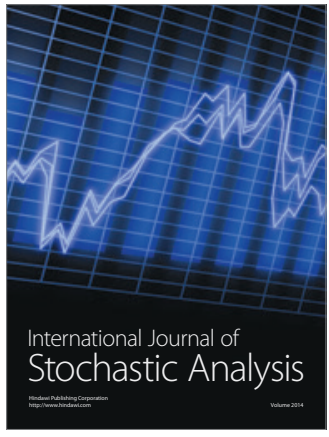

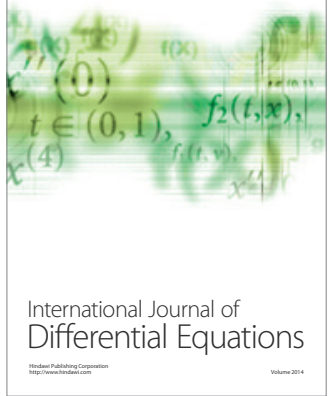
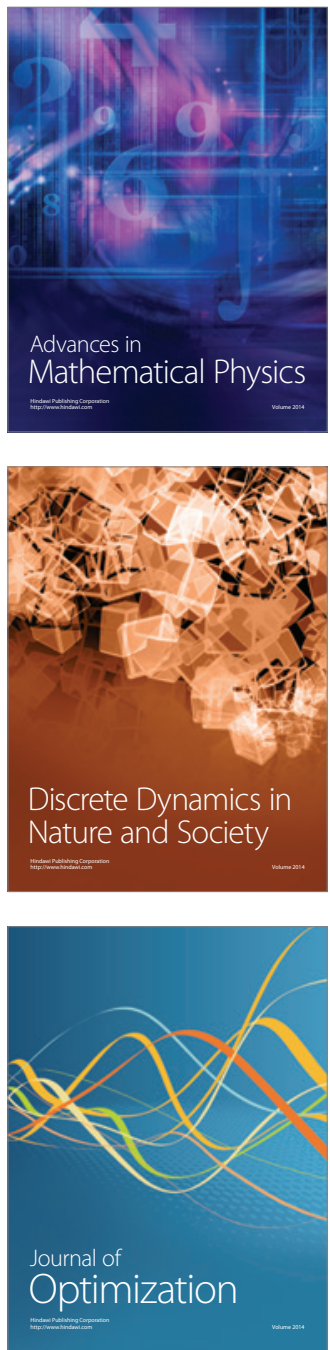